\title{
Structure of Normal Alkane Evaporated Films: Molecular Orientation
}

Koji Nozaki ${ }^{*}$, Rieko Saihara, Kiyoshi Ishikawa, and Takashi Yamamoto Department of Physics, Graduate School of Science and Engineering, Yamaguchi University, Yamaguchi 753-8512, Japan

The molecular orientations of $n$-alkane $\left(n-\mathrm{C}_{23} \mathrm{H}_{48}, n-\mathrm{C}_{24} \mathrm{H}_{50}, n-\mathrm{C}_{25} \mathrm{H}_{52}, n-\mathrm{C}_{26} \mathrm{H}_{54}\right.$ and $n-\mathrm{C}_{27} \mathrm{H}_{56}$ ) evaporated films prepared by considering various deposition conditions, including the type of substrate, substrate temperature, and evaporation rate, have been systematically investigated by X-ray diffraction analysis and scanning probe microscopy. Two typical molecular orientation states, namely, the "perpendicular orientation state", in which the molecular chain axes are perpendicular to the substrate surface, and the "parallel orientation state", in which the molecular chain axes are parallel to the substrate surface, are observed. At a high substrate temperature and a low evaporation rate, a "perpendicular (P) film", which consists of only the perpendicular orientation state, is obtained. At a low substrate temperature and a high evaporation rate, a "coexistent (C) film", which consists of both the perpendicular and parallel orientation states, is obtained. It is concluded, from the experimental results, that the most thermodynamically stable is the perpendicular orientation state. Furthermore, it is found that the molecular orientation behavior depends on the type of substrate and deposition time. These molecular orientation dependences are considered to be mainly due to a kinetic mechanism rather than the thermodynamic stability in the case of the evaporated film in this study.

KEYWORDS: $n$-alkane, evaporated film, PVD, molecular orientation, X-ray diffraction, SPM

*E-mail address: nozaki@yamaguchi-u.ac.jp 


\section{Introduction}

There have been many studies on organic molecular thin films owing to the fundamental science interest as well as their industrial applications in recent years. In a thin film system, a large size anisotropy exists, i.e., the thin film system has a large surface area or large interface area with a substrate. Interesting phenomena, which are due to the size effect and peculiar to the thin film system, are often observed. This size anisotropy also affects the structure formation, which dominates the physical properties of the thin film system.

Long hydrocarbon chains are the fundamental frames of many organic molecules, such as lipids, oils, fats, and polymers. Therefore, hydrocarbon plays an important role in structure formation process, such as the crystallizations of fats and polymers, and the formations of lipid membranes. The arrangement of hydrocarbon chains in organic materials affects the physical properties and functions. Therefore, it is very important to elucidate the biomembrane function or industrial application of the materials to understand such an arrangement. Many studies, which aimed at the fundamental understanding of the structure formation of hydrocarbons, have been performed using a simple long hydrocarbon molecule, such as $n$-alkane. On the basis of condensation structure of long hydrocarbon chains, it also became clear that similarity exists in various systems ${ }^{1,2}$. Long hydrocarbon molecules are arranged in an ordered lamellar structure, in which the molecular chain axes are parallel to each other and their end groups are on the same plane. In a crystal of a long-chain hydrocarbon molecule, the lamellae stack in layers with a three-dimensional (3D) periodicity. On the other hand, the monolayer and bilayer systems are ultrathin films consisting of one lamella and two lamellae, respectively.

Surface freezing ${ }^{3,4)}$ is often observed as a characteristic surface phenomenon in long-chain hydrocarbon molecular systems. An unconventional temperature dependence for the surface tension of bulk $n$-alkane liquid was first observed at a temperature above the bulk melting point. ${ }^{3)}$ The result of the surface tension measurement led to the discovery of surface freezing. The unconventional surface tension behavior was considered to be due to the formation of the surface monolayer in which molecular chain axes are almost perpendicular to a bulk free surface (interface between bulk liquid and air) at a temperature above the bulk melting point. The formation of the surface monolayer was also confirmed by X-ray diffraction analysis and X-ray reflectivity measurement. ${ }^{4)}$ Such an ordered anisotropic molecular arrangement in an $n$-alkane monolayer was also observed in thin film system on a solid substrate, such as $\mathrm{SiO}_{2}{ }^{5}$ ) Many studies on the formation of the surface monolayer in an $n$-alkane thin film system 
on a solid substrate $\left(\mathrm{SiO}_{2}\right)$ have been performed by various experimental techniques since then. ${ }^{6-14)}$

A thin film with a long chain of hydrocarbon molecule is also used as a model system for an organic molecular thin film. Many studies have been performed for many years using the hydrocarbon molecular film prepared by physical vapor deposition methods, such as vacuum evaporation. In an evaporated film system with a long chain of hydrocarbon molecules, two typically preferred molecular orientation states were observed, as shown in Fig. 1. One is the "perpendicular orientation state", in which molecules appear with their chain axes perpendicular to the substrate surface, and the other is the "parallel orientation state", in which molecular chain axes are parallel to the substrate surface. ${ }^{15,16)}$ The molecular orientation behavior depends on preparation conditions. In a fatty acid evaporated film, ${ }^{15)}$ the perpendicular orientation state tends to appear when the deposition rate is low or the substrate temperature is high. The dependence of the type of substrate on the molecular orientation was also confirmed. It is important to reveal the mechanism of the formation of the characteristic molecular orientation not only for basic science fields but also for the application of the organic thin film, particularly from the aspect of effective functional generation by controlling the molecular orientation.

Ueda and Ashida reported that the molecular orientation of an $n$-alkane evaporated film is markedly affected by substrate temperature. ${ }^{16)}$ In the parallel orientation state of the $n$-alkane evaporated film, the molecules change their orientation state to the perpendicular orientation state during annealing at a temperature below the melting point. ${ }^{17,18)}$ Fukao, and coworkers measured the orientational distribution of molecular chain axes in an $n$-alkane evaporated film by energy-dispersive $X$-ray diffraction analysis and suggested that in the perpendicular orientation state, $n$-alkane molecules orient their chain axes within $0.1^{\circ}$ around the normal surface to the substrate surface. ${ }^{18,19)}$ Furthermore, the change in molecular orientation during annealing is irreversible and a few molecules are considered to turn cooperatively on the basis of the measured temperature dependence of the rate of molecular orientation change.

In this study, we focus on two typical molecular orientations, namely, the perpendicular and parallel orientations, in $n$-alkane evaporated films. The molecular orientations of such films prepared under various conditions are investigated. The mechanism of the formation of a characteristic molecular orientation is discussed.

An $n$-alkane crystal has a low-temperature ordered phase (LO phase) and a characteristic high-temperature phase, which is called the rotator phase ( $\mathrm{R}$ phase). ${ }^{1,20,21)}$ 
In the LO phase, $n$-alkane molecules are fully extended taking all-trans conformations and registered in layers, in which molecular chain axes are parallel to each other. The molecules have a long-range order with respect to the orientation around their long axes as well as their mass position center. Normal alkane shows polymorphism depending on its carbon number $n$. For $n$-alkanes with an odd carbon number, the lowest temperature ordered phase is an orthorhombic phase ( $\mathrm{O}$ phase). Side packing is of the herringbone type and the molecular axes are perpendicular to the layer surface. ${ }^{22)}$ For $n$-alkanes with an even carbon number, two modifications are found to correspond to the lowest temperature ordered phase: a triclinic phase (T phase) with $n_{\mathrm{even}} \leq 26$ and a monoclinic phase (M phase) with $n_{\text {even }}>26$; the molecules tilt from the normal surface to the layer surface in the T and O phases. ${ }^{23,24)}$ Normal alkanes with $9 \leq n_{\text {odd }} \leq 39$ and $20 \leq n_{\text {even }}$ $\leq 38$ have an $\mathrm{R}$ phase just below the melting point. In this $\mathrm{R}$ phase, the molecules still have a three-dimensional long-range positional order, but rack the long-range order in their orientation. Despite their disordered structure, the $\mathrm{R}$ phase is indeed a crystalline state. The molecules in the $\mathrm{R}$ phase produce an active thermal motion and have a high mobility. In particular, the translation and rotation along the chain axes are excited. ${ }^{25-32}$ ) Recent X-ray investigations have shown the presence of five $\mathrm{R}$ phases with different crystal structures. Although they are in solid state, the long-range diffusion of molecules in the $\mathrm{R}$ phase was confirmed by X-ray diffraction analysis ${ }^{33)}$ and optical microscopy. ${ }^{34,35)}$ These characteristics are expected to be relate to the various properties of biological membranes constructed by lipids. Hence, in recent years, there has been a growing interest in the $\mathrm{R}$ phase and its physical properties.

\section{Experimental}

Five $n$-alkanes $\left(n-\mathrm{C}_{n} \mathrm{H}_{2 n+2}\right.$ : abbreviated $\left.\mathrm{Cn}\right), n$-tricosane $(\mathrm{C} 23), n$-tetracosane (C24), n-pentacosane (C25), n-hexacosane (C26), and $n$-heptacosane (C27), were purchased from Tokyo Kasei Kogyo; the LO-R transition points $\left(T_{\mathrm{LO}-\mathrm{R}}\right)$, melting points $\left(T_{\mathrm{m}}\right)$, lowest temperature ordered phase, and purities are listed in Table I. No further purification of the samples purchased was carried out.

Three types of substrate, namely, polyimide, glass, and silicon (abbreviated PI, GL, and SI, respectively), were used for deposition. The polyimide film (UPILEX, 0.03 $\mathrm{mm}$ in thickness) produced by Ube Industries, Ltd. was cut into pieces $18 \times 18 \mathrm{~mm}^{2}$ in size for use as the PI substrate. A $\mathrm{Si}(100)$ wafer $(0.37 \mathrm{~mm}$ in thickness) was cut into pieces of the same size as other substrates and used for the SI substrate. Cover glass plates made of soda glass $\left(18 \times 18 \mathrm{~mm}^{2}, 0.15 \mathrm{~mm}\right.$ in thickness $)$ were purchased from 
Natsunami Glass Industries and used as the GL substrate.

Vacuum evaporation was performed using a standard apparatus (ULVAC, PVC160). The $n$-alkane sample was evaporated from a tungsten boat and deposited on a substrate mounted on a metal block with an electric heater. The evaporation rate was regulated by controlling the evaporation temperature, which was regulated by controlling the electric current directly passing through the tungsten boat. The temperatures of the tungsten boat and the substrate were controlled using PID controllers within \pm 0.5 and $\pm 1^{\circ} \mathrm{C}$, respectively, where K-type thermocouples were attached to the boat and substrate. Vacuum evaporation was performed at a pressure of $2 \times 10^{-3} \mathrm{~Pa}$. During deposition, the evaporation rate and film thickness are monitored using a standard deposition monitor (ULVAC CRTM-6000) with a quartz crystal oscillator.

The molecular orientation of the $n$-alkane evaporated film was investigated by $\mathrm{X}$-ray diffraction analysis using a standard diffractometer (Rigaku RADIIA) with a symmetrical reflection method, in which $\mathrm{Cu}-\mathrm{K} \alpha$ radiation was used. The sample evaporated film with the substrate was attached to a sample holder, so that only Bragg reflections, the scattering vectors of which were perpendicular to the substrate surface, were observable.

The surface morphology of the evaporated film was observed by using a scanning probe microscope (SPM; SII Nanotechnology, SPI3800N). The dynamic force mode (DFM) was applied, and the scanning area used was $10 \times 10 \mu \mathrm{m}^{2}$.

\section{Results}

\section{1 $X$-ray diffraction}

Figures 2(a)-2(d) show the X-ray diffraction patterns of C25 evaporated films on PI substrates prepared under various deposition conditions, and Fig. 2(e) shows the X-ray diffraction pattern of $\mathrm{C} 23$ isotropic powder. In Fig. 2(e), reflections at angles smaller than $15^{\circ}$ correspond to the $00 \mathrm{l}$ reflections from the lamellar structure. Two strong reflections around $2 \theta=21^{\circ}$ and $24^{\circ}$ are the $110_{\text {s }}$ and $200_{\text {s }}$ reflections from the side packing of molecules, respectively, where subscript "s" indicates the subcell of the C-C-C unit; Bragg reflections on the same row line near the equator, $110,111,112, \ldots$, cannot be resolved because of the short reciprocal axis $c^{*}$. In Fig. 2(a), only the 00l reflections are observed. This result indicates that the $(00 l)$ planes are parallel to the substrate surface. In such a situation, molecular chain axes are perpendicular to the substrate surface in the case of odd $n$-alkanes (orthorhombic structures), i.e., the 
evaporate film consists of only domains in the perpendicular orientation states. Here, we call this type of film a "perpendicular (P) film". The observed very sharp reflection peak shows the highly preferred molecular orientation of the films. In the case of even $n$-alkanes (triclinic or monoclinic structures), although molecular chain axes tilt from the normal plane to the (001) plane, we call the state, in which the (001) planes are parallel to a substrate, the perpendicular orientation state. In Figs. 2(b)-2(d), the 110 s reflection is observed as well as $00 \mathrm{l}$ reflections. Therefore, the film consists of domains in both the perpendicular and parallel orientation states. In the latter case, the $\left(110_{\mathrm{s}}\right)$ plane is parallel to the substrate surface, because other side packing reflections, such as $200_{\text {s }}$ reflection, are not observed. We call this type of film a "coexistent (C) film".

The molecular orientation behaviors of the $n$-alkane evaporated films prepared under various deposition conditions were investigated by X-ray diffraction analysis. Those of the films deposited on the PI substrate are summarized in Fig. 3 as functions of evaporation rate $\left(v_{\mathrm{e}}\right)$ and substrate temperature $\left(T_{\mathrm{s}}\right)$. Figures 3(a)-3(e) show the molecular orientation behaviors of the evaporated films on the PI substrate for the five $n$-alkanes investigated here. The vertical axis corresponds to the degree of supercooling, $\Delta T \equiv T_{\mathrm{s}}-T_{\mathrm{m}}$, where $T_{\mathrm{m}}$ is the melting temperature of the $n$-alkane sample. The horizontal axis shows the evaporation rate. When the evaporation rate is low or the substrate temperature is high, the $\mathrm{P}$ film tends to be prepared. This tendency is significant when the substrate temperature is in the $\mathrm{R}$ phase region of the $n$-alkane sample used here. On the other hand, at a low substrate temperature and a high evaporation rate, the parallel orientation state coexists with the perpendicular orientation state for all $n$-alkane samples, and a $\mathrm{C}$ film is formed. It seems that the deposition condition of the $\mathrm{P}$ film in the case of even $n$-alkanes is wider than that of the $\mathrm{P}$ film in the case of odd $n$-alkanes. This may be due to the fact that different polymorphs appear between the even ( $T$ phase) and odd (O phase) $n$-alkane evaporated films. In the case of odd $n$-alkane evaporated films, the deposition condition of the P film is wide for a short $n$-alkane (small carbon number). Thus, the carbon number dependence of the molecular orientation behavior of the $n$-alkane evaporate film is observed.

Figures 4(a)-4(e) show the molecular orientations of the $n$-alkane evaporated films on the GL substrate. The P film also tends to be formed at a low evaporation rate or at a high substrate temperature in the case of using the GL substrate. However, the deposition condition of the P film on the GL substrate is clearly narrower than that of the P film on the PI substrate. It is confirmed that the molecular orientation behavior depends on the type of substrate. This will be discussed later. 


\section{2 Morphology}

Figures 5(a) and 5(b) show the SPM (DFM) images of the surface of the C23 P film on the SI substrate. In the surface image in Fig. 5(a), the stacked flat terraces are observed. In the cross-sectional image in Fig. 5(b), the step height of the stacked flat terraces is estimated to be about $3.1 \mathrm{~nm}$, which corresponds to the chain length of the C23 molecule in the extended all-trans conformation. A schematic of the surface morphology (cross-sectional image) is predicted from the SPM image and shown in Fig. 5(c). The monolayer, in which the molecules are arranged with their molecular chain axes being parallel to each other, stacks in layers in the growth direction of the film. In the SPM image, the monolayer is observed to exhibit the terrace morphology.

Figures 6(a) and 6(b) show the SPM images of the surface of the C23 C film on the SI substrate. The stacked terraces are also observed in the surface image of the $\mathrm{C}$ film [Fig. 6(a)]. The terrace area is considered to correspond to the perpendicular orientation state area. On the other hand, square rods on the surface are observed in the flat terrace area. Such square rods have never been observed in the SPM image of the P film, for which no side packing reflection is observed in the X-ray diffraction profile. Therefore, it can be concluded that square rods correspond to domains in the parallel orientation state. In the cross-sectional image [Fig. 6(b)], the width and height of the rods are about 500-600 $\mathrm{nm}$. The surface of the $\mathrm{C}$ film is rougher than that of the $\mathrm{P}$ film. With the relative intensity of the side packing reflection $\left(110_{\mathrm{s}}\right.$ reflection) in the X-ray diffraction profile, the square rod area in the surface image relatively increases. This fact is consistent with the above-mentioned conclusion.

\subsection{Deposition time dependence of the molecular orientation}

Figures 7(a) and 7(b) show the deposition time dependences of the X-ray diffraction profiles of the $\mathrm{C} 23$ evaporated films deposited on the SI substrate at $25^{\circ} \mathrm{C}$. At an evaporation rate of $25 \mathrm{mg} / \mathrm{h}$ [Fig. 7(a)], the $110_{\mathrm{s}}$ reflection is not observed at $2 \mathrm{~min}$, and a P film is formed. In the SPM image of the same film, the square rod area, which is peculiar to the $\mathrm{C}$ film, is not observed. With deposition time, the $110_{\mathrm{s}}$ reflection intensity increases; thus, such a reflection becomes observable. At the initial stage of deposition, the formation of the perpendicular orientation state is dominant. On the

other hand, with an increase in deposition time, i.e., an increase in film thickness, the formation of the parallel orientation state accelerates. The significant deposition time dependence of the molecular orientation behavior is confirmed. At an evaporation rate of $42 \mathrm{mg} / \mathrm{h}$, the $110_{\mathrm{s}}$ reflection is observed even at $2 \mathrm{~min}$. In this case, the early stage of deposition may occur rapidly and cannot be observed even at $2 \mathrm{~min}$ owing to high 
evaporation rate. The dominant growth mode changes from the growth of the perpendicular orientation state to that of the parallel orientation state with time, or with an increase in film thickness. Incidentally, only the P film is obtained using the PI substrate under the same deposition condition.

\subsection{Substrate dependence of molecular orientation}

It has been clarified that the molecular orientation depends on the type of substrate and deposition time. It can easily be considered that the origin of the substrate dependence of the molecular orientation is related to the direct interaction between the $n$-alkane molecule and the substrate. To verify this consideration, two films were prepared by the following method and their molecular orientations were investigated. First, P films of $100 \mathrm{~nm}$ in thickness were prepared on the PI and SI substrates. Then, C23 were deposited on these P film substrates successively under the same conditions as those in Fig. 7(a), where the evaporation rate was $25 \mathrm{mg} / \mathrm{h}$. The second evaporation was performed when the direct interaction between the substrate and the C23 molecules was completely inhibited, and the characteristics of the base substrates were screened out. The X-ray diffraction profiles of the films prepared above are shown in Fig. 8. During the deposition of $\mathrm{C} 23$ on a PI-based P film, a P film is formed. On the other hand, a $\mathrm{C}$ film grows on an SI-based $\mathrm{P}$ film. These results show that the substrate dependence of the molecular orientation exhibits even when the direct interaction between the substrate and the $n$-alkane molecules is completely masked. This substrate dependence seems not to be caused by the direct interaction between the substrate and the molecules.

\section{Discussion}

The conditions suitable for the formation of the $\mathrm{P}$ film are a low evaporation rate and a high substrate temperature. Furthermore, when the prepared $\mathrm{C}$ film is annealed at a temperature below the melting point, it changes to a $\mathrm{P}$ film irreversibly. ${ }^{17,18)}$ From the obtained experimental results, it is easily concluded that the most thermodynamically stable state is the perpendicular orientation state. Actually, when the $n$-alkane liquid is slowly cooled from the melt and solidified after being thinly spread on the substrate, the P film is prepared. This fact is very consistent with the above-mentioned conclusion. The $\mathrm{P}$ film formation tendency in the case of short $n$-alkanes is more significant than that in the case of long $n$-alkanes. Because of the high mobility of short chains, the system easily shifts to the most thermodynamically stable 
perpendicular orientation state. Since the molecules also have a high mobility in the $\mathrm{R}$ phase, the P film is more likely to be prepared.

If the perpendicular orientation state is thermodynamically stable, the appearance of the parallel orientation state is caused by kinetic mechanism. After the molecules in the vapor phase adsorb on the substrate surface, they gather together by diffusion on the substrate surface, resulting in the nucleation and growth of the crystallites in the film. In the case that an isolated molecule adsorbs on the substrate surface, the molecule is expected to settle parallel to the substrate surface due to the interaction between a single hydrocarbon chain and the substrate. At a high substrate temperature, since the molecules have a high mobility, the deposited molecules in the parallel orientation state may aggregate and nucleate. The primary nucleation rate at a high temperature is considered to be relatively low. Hence, the primary nuclei are in the perpendicular orientation state, which is the most stable state in aggregation systems. Generally, the growth rate of the $n$-alkane O phase crystal is higher in the $\left\langle 110_{\mathrm{s}}\right\rangle$ direction than in other directions. Therefore, immediately after the primary nucleation of the perpendicular orientation state, the crystal grows fast in the $\left\langle 110_{\mathrm{s}}\right\rangle$ direction parallel to the substrate surface and covers a large area on the substrate. When the crystal grows in the $<001>$ direction, the secondary nucleation on the (001) crystal surface is required. Hence, the growth rate in the $<001>$ direction is very low, indicating layer-by-layer growth. As a result, the surface of the P film is very smooth; only the steps corresponding to once or twice the molecular length appear on the surface (Fig. 5). On the other hand, at a low substrate temperature and a high evaporation rate, the molecules have a low mobility, and the molecules that adsorb on the substrate surface in parallel gather locally. The aggregated molecules that cannot climb over the potential barrier remain in the meta-stable parallel orientation state and become the nuclei of the parallel orientation state. Furthermore, many molecules are supplied one after another and are imported into the nuclei, resulting in the $<110_{\mathrm{s}}>$ crystal growth. As a result, the formation of the parallel orientation state becomes dominant. In this situation, since the primary nucleation rate is relatively high, multinucleation occurs on the substrate. The respective primary nuclei grow fast in the $\left\langle 110_{\mathrm{s}}>\right.$ direction individually, and the surface seems to become rough (Fig. 6).

Generally, the primary nucleation on the substrate surface is considered to be controlled by the direct interaction between the molecules and the substrate. As the interaction is altered by the type of substrate, it can easily be considered that the molecular orientation varies with the type of substrate. However, the observed substrate dependence on the molecular orientation seems to be caused by a kinetic mechanism as 
well as the molecular interaction under the deposition conditions of this work. In the vacuum evaporation method, the diffusion of the kinetic energy of the molecules as heat through the substrate after adsorption plays an important role in the film formation. When the molecules in the vapor phase adsorb on the PI substrate, the kinetic energy of the molecules cannot diffuse owing to the low thermal conductivity and low heat capacity of the thin polyimide substrate. In this case, since the molecules still have a high mobility, they fall down in the stable state through slow cooling and nucleate in the perpendicular orientation state. Therefore, the P film tends to form on the PI substrate. In the case of using relatively thick SI and GL substrates, the kinetic energy of the molecules is absorbed by the substrate in a short time because of the relatively high thermal conductivity and high heat capacity. Therefore, $\mathrm{C}$ film formation tendency is more significant than that in the case of using a thin PI substrate.

The above-mentioned speculation well elucidates the time dependence of the molecular orientation in Fig. 7. The total heat capacities of the substrate and film deposited vary with an increase in film thickness during evaporation. Furthermore, their thermal conductivities also vary. Therefore, the kinetic mechanism in the growth of the film is variable. The deposition time dependence of the molecular orientation is considered to be caused by the change in the type of kinetic mechanism in the growth of the film. This consideration also well explains the experimental result shown in Fig. 8. Because the heat capacity and/or the thermal conductivity varies between the PI-based and SI-based P films, the molecular orientation behaviors of the two cases are different.

\section{Conclusions}

We have systematically investigated the molecular orientations of the $n$-alkane evaporated films prepared under various deposition conditions by X-ray diffraction analysis and SPM. The major results are summarized as follows.

Two typical molecular orientation states, namely, the perpendicular and parallel orientation states, appear in the $n$-alkane evaporate films. At a high substrate temperature and a low evaporation rate, the $\mathrm{P}$ film consisting of only the perpendicular orientation state is obtained. At a low substrate temperature and a high evaporation rate, the $\mathrm{C}$ film consisting of both the perpendicular and parallel orientation states is obtained. It is concluded, from the experimental results, that the most thermodynamically stable state is the perpendicular orientation state. Furthermore, it has been clarified that the molecular orientation behavior depends on the type of substrate and deposition time. These molecular orientation dependences are considered to be mainly caused by a 
kinetic mechanism in the case of the evaporated film in this study. The direct interaction between the $n$-alkane molecule and the substrate is not effective for the molecular orientation.

\section{Acknowledgment}

This work was supported by a Grant-in-Aid for Scientific Research (No. 14540378) from the Ministry of Education, Culture, Sports, Science and Technology. 
1) B. M. Craven, Y. Lange, G. G. Shipley, and J. Steiner: in Handbook of Lipid Research, ed. D. M. Small (Plenum, New York, 1986), Vol. 4.

2) E. B. Sirota: Langmuir 13 (1997) 3849.

3) J. C. Eanshaw and C. J. Hughes: Phys. Rev. A 46 (1992) R4494.

4) X. Z. Wu, E. B. Sirota, S. K. Shinha, B. M. Ocko, and M. Deutsch: Phys. Rev. Lett. 70 (1993) 958.

5) C. Merkl, T. Pfohl, and H. Reigler: Phys. Rev. Lett. 79 (1997) 4625.

6) A. Holtzwarth, S. Leporatti, and H. Riegler: Europhys. Lett. 52 (2000) 623.

7) Schollmeyer, B. Ocko, and H. Riegler: Langmuir 18 (2002) 4351.

8) H. Schollmeyer, B. Struth, and H. Riegler: Langmuir 19 (2003) 5042.

9) P. Lazar, H. Schollmeyer, and H. Riegler: Phys. Rev. Lett. 94 (2005) 116101.

10) U. G. Volkmann, M. Pino, L. A. Altamirano, H. Taub, and F. Y. Hansen: J. Chem. Phys. 116 (2002) 2107.

11) H. Mo, H. Taub, U. G. Volkmann, M. Pino, S. N. Ehrlich, F. Y. Hansen, E. Lu, and P. Miceli: Chem. Phys. Lett. 377 (2003) 99.

12) H. Mo, S. Trogisch, H. Tab, S. N. Ehrlich, U. G. Volkmann, F. Y. Hansen, and M. Pino: Phys. Status Solidi A 201 (2004) 2375.

13) Mo, S. Trogisch, H. Tab, S. N. Ehrlich, U. G. Volkmann, F. Y. Hansen, and M. Pino: J. Phys. Condens. Matter 16 (2004) S2905.

14) F. Kruchten, K. Knorr, U. G. Volkmann, H. Taub, F. Y. Hansen, B. Matthies, and K. W. Herwig: Langmuir 21 (2005) 7507.

15) K. Sato, H. Takiguchi, S. Ueno, J. Yano, and K. Yase: in Advances in the Understanding of Crystal Growth Mechanisms, ed. T. Nishinaga, K. Nishioka, J. Harada, A. Sasaki, and H. Takei (Elsevier Science, Amsterdam 1997) PP 349.

16) K. Ueda and M. Ashida: J. Electron Micros. 29 (1980) 38.

17) K. Tanaka, S. Kimura, J. Nakahashi, S. Umemoto, N. Okui, and T. Sasaki: Kobunshi Ronbunshu 44 (1987) 817 [in Japanese].

18) K. Fukao, T. Horiuchi, and K. Matsushige: Thin Solid Films 171 (1989) 359.

19) K. Fukao: Thin Solid Films 197 (1991) 157.

20) M. G. Broadhurst: J. Res. Natl. Bur. Stand., Sect. A 66 (1962) 241.

21) A. Müller: Proc. R. Soc. London Ser. A 138 (1932) 514.

22) A. E. Smith: J. Chem. Phys. 21 (1953) 2229.

23) A. Müller and K. Lonsdale: Acta Crystallogr. 1 (1948) 129.

24) H. M. M. Shearer and V. Vand: Acta Crystallogr. 9 (1956) 379.

25) J. Doucet, I. Denicolo, and A. Graievich: J. Chem. Phys. 75 (1981) 1523.

26) J. Doucet, I. Deniclo, A. Graievich, and A. Collet: J. Chem. Phys. 75 (1981) 5125. 
27) I. Denicolo, J. Doucet, and A. F. Graievich: J. Chem. Phys. 78 (1983) 1465.

28) J. Doucet, I. Denicolo, A. F. Graievich, and C. Germain: J. Chem. Phys. 80 (1984) 1647.

29) G. Ungar: J. Phys. Chem. 87 (1983) 689.

30) G. Ungar and N. Maŭić: J. Phys. Chem. 89 (1985) 1036.

31) E. B. Sirota, H. E. King, Jr., D. M. Singer, and H. H. Shao: J. Chem. Phys. 98 (1993) 5809.

32) E. B. Sirota and D. M. Singer: J. Chem. Phys. 101 (1996) 10873.

33) T. Yamamoto and K. Nozaki: Polymer 35 (1994) 3340.

34) T. Yamamoto and K. Nozaki: Polymer 36 (1995) 2505.

35) T. Yamamoto, T. Aoki, S. Miyaji, and K. Nozaki: Polymer 38 (1997) 2643.

Table I. Normal alkane samples used in this work.

\begin{tabular}{cclcc}
\hline$n$-Alkane & $T_{\text {LO-R }}\left({ }^{\circ} \mathrm{C}\right)$ & \multicolumn{1}{l}{$T_{\mathrm{m}}\left({ }^{\circ} \mathrm{C}\right)$} & Lowest temperature phase & Purity $(\mathrm{wt} \%)$ \\
\hline $\mathrm{C} 23$ & 41.0 & 47.5 & $\mathrm{O}$ & $98<$ \\
$\mathrm{C} 24$ & 47.5 & 50.9 & $\mathrm{~T}$ & $99<$ \\
$\mathrm{C} 25$ & 47.8 & 54.0 & $\mathrm{O}$ & $98<$ \\
$\mathrm{C} 26$ & 52.5 & 56.4 & $\mathrm{~T}$ & $97<$ \\
$\mathrm{C} 27$ & 51.6 & 58.5 & $\mathrm{O}$ & $98<$ \\
\hline
\end{tabular}




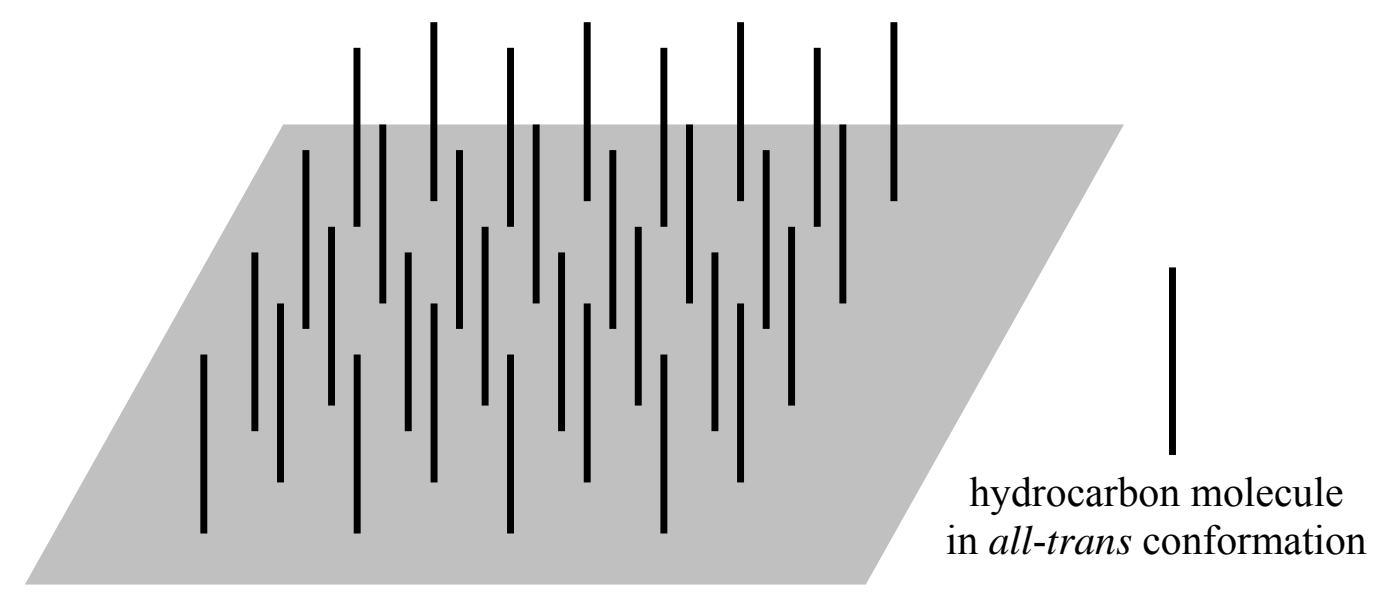

perpendicular orientation state

(a)

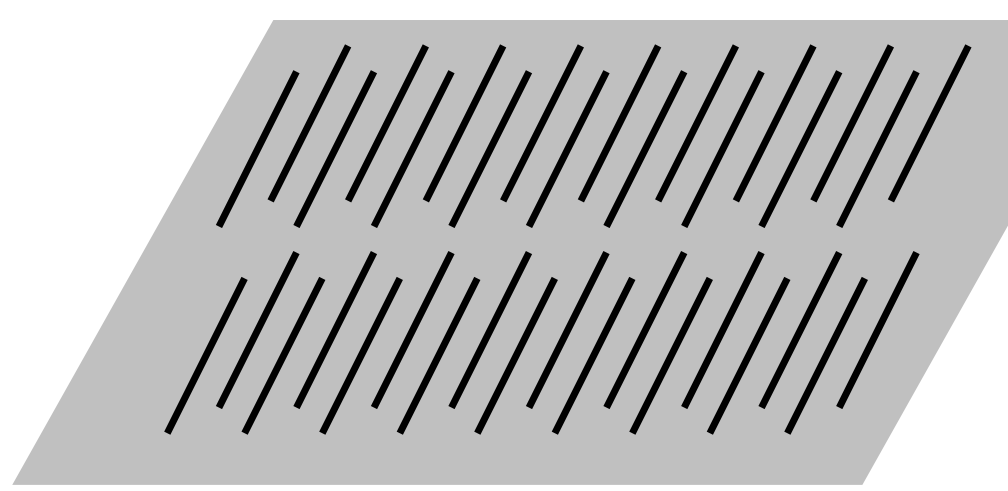

parallel orientation state

(b)

Figure 1 Two typically preferred molecular orientation states, (a) perpendicular and (b) parallel orientation states, appearing in evaporated film with long-chain molecules. 


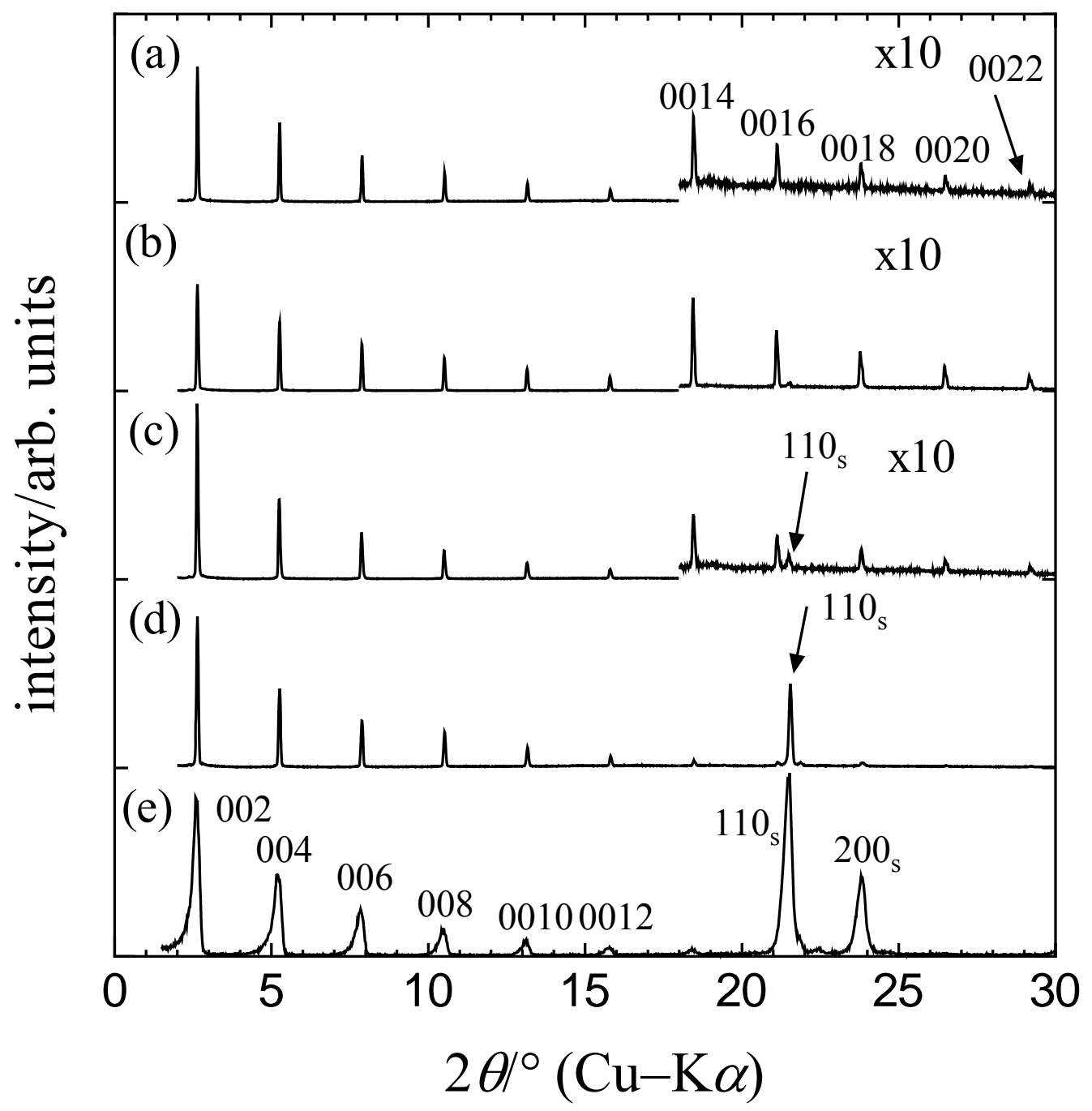

Figure 2 X-ray diffraction patterns of C25 evaporated films on PI substrates prepared under various deposition conditions [(a)-(d)]. To compare them, the pattern of C25 isotropic powder is shown (e). The reflections at angles smaller than $15^{\circ}$ correspond to the $00 \mathrm{l}$ reflections from the lamellar structure. The reflection at $2 \theta=21.4^{\circ}$ is the $110 \mathrm{~s}$ reflection from the side packing of molecules. The pattern showing only $00 \mathrm{l}$ reflections corresponds to the film consisting of only the perpendicular orientation state, i.e., the $\mathrm{P}$ film. 


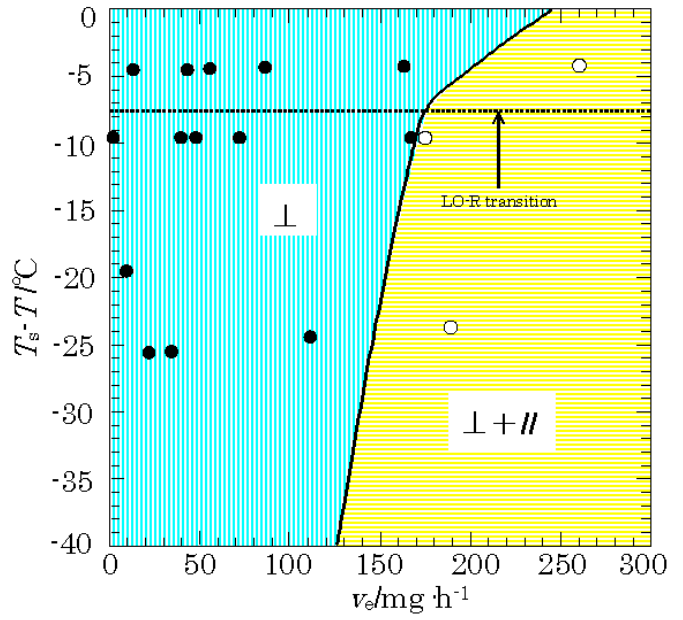

(a)

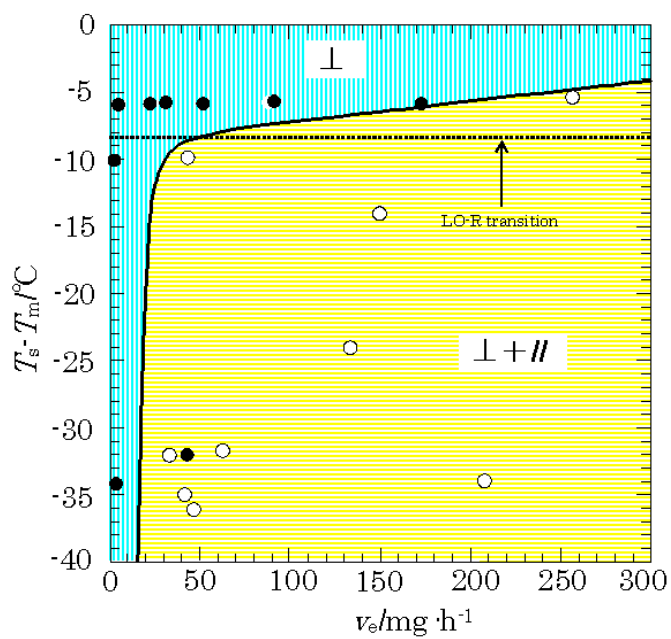

(c)

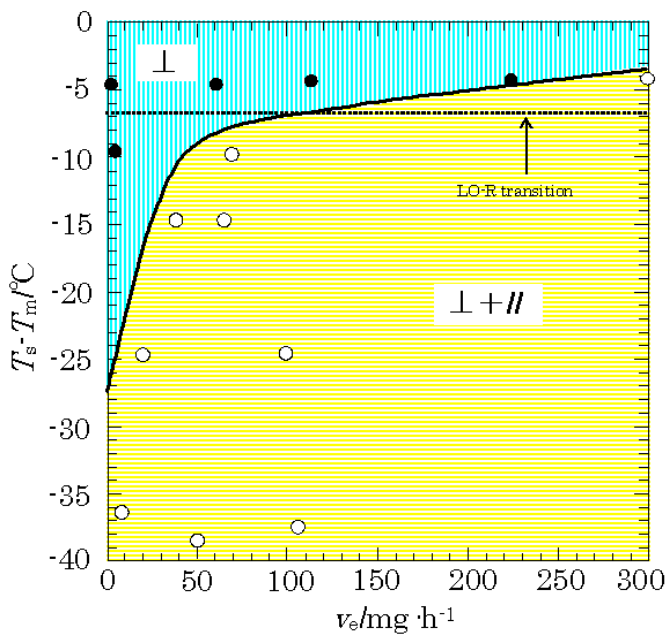

(e)

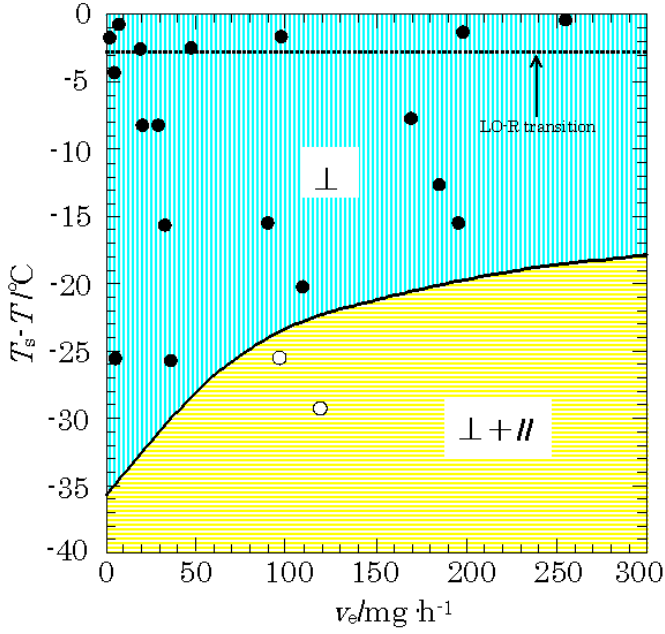

(b)

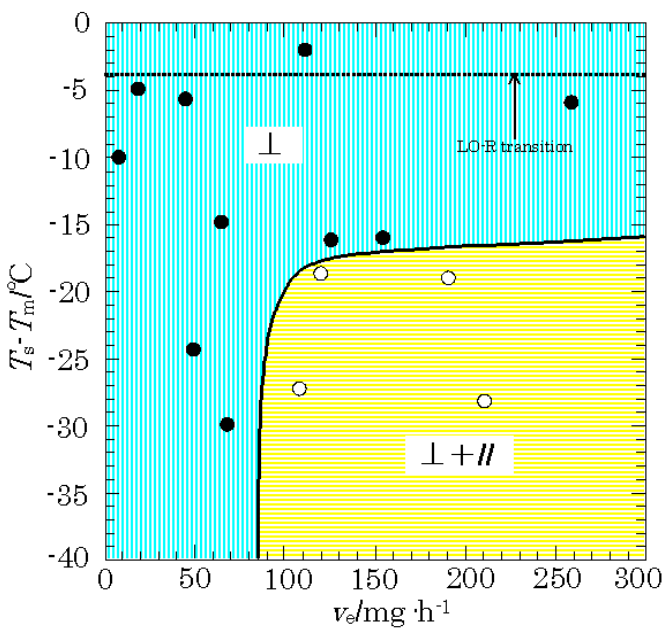

(d)

Figure 3 Molecular orientation behaviors of (a) C23, (b) C24, (c) C25, (d) C26, and (e) $\mathrm{C} 27$ evaporated films deposited on PI substrate. The vertical axis corresponds to $\Delta T=T_{\mathrm{s}}-T_{\mathrm{m}}$, where $T_{\mathrm{s}}$ is the substrate temperature and $T_{\mathrm{m}}$ is the melting point of the deposited $n$-alkane sample. Full and open circles represent the deposition conditions under which the $\mathrm{P}$ and $\mathrm{C}$ films are obtained, respectively. 


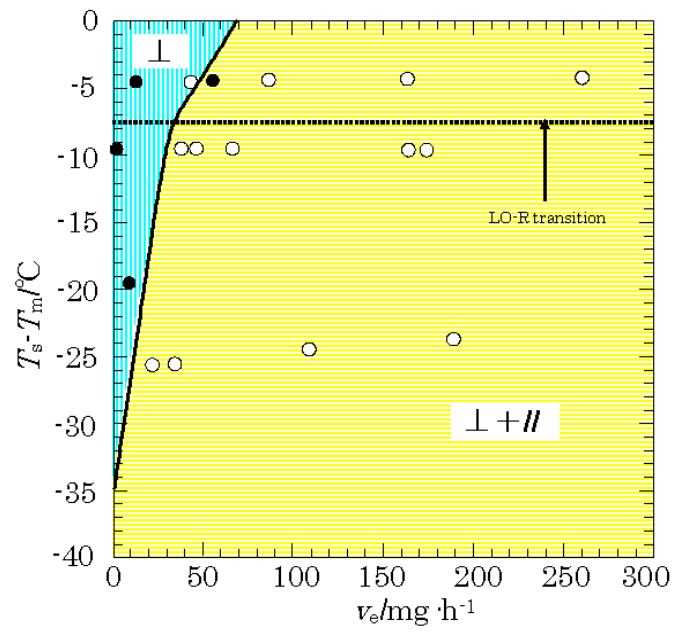

(a)

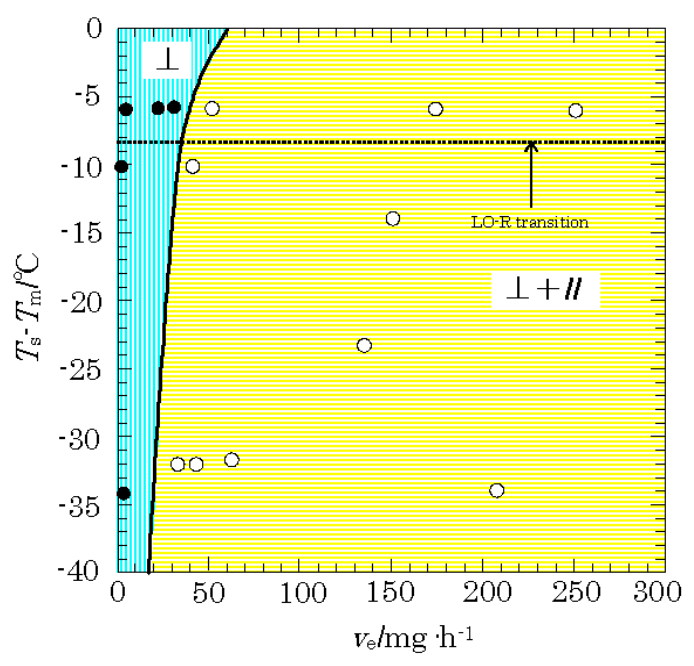

(c)

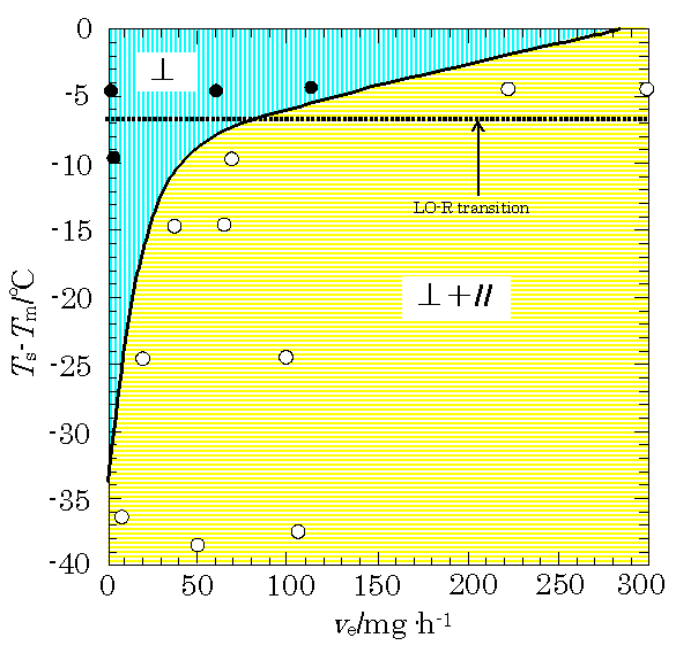

(e)

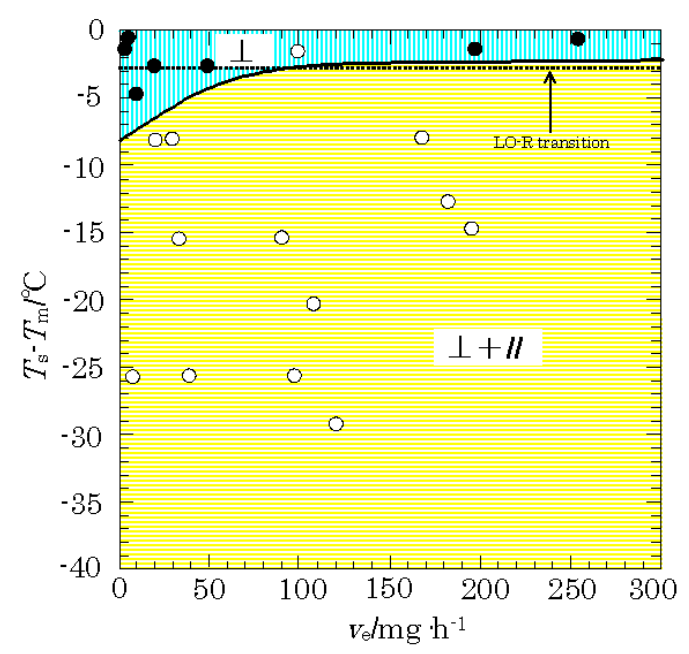

(b)

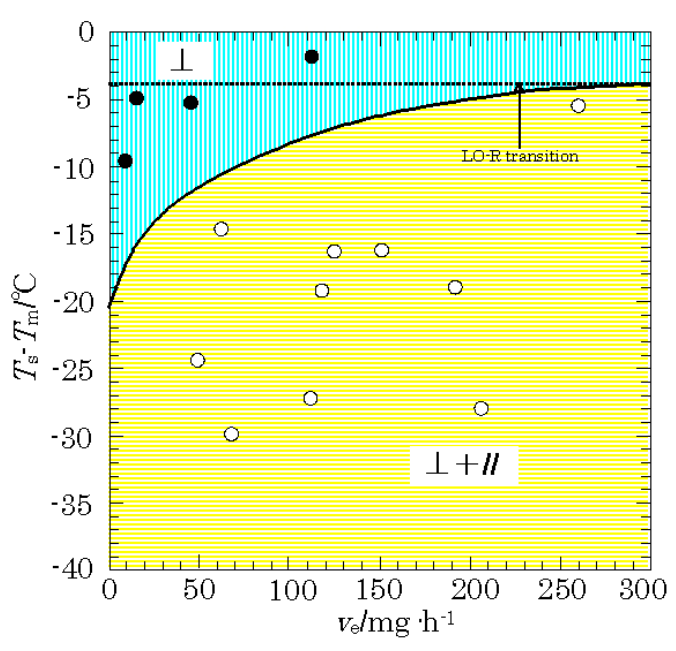

(d)

Figure 4 Molecular orientation behaviors of (a) C23, (b) C24, (c) C25, (d) C26, and (e) C27 evaporated films deposited on GL substrate. Full and open circles represent the deposition conditions under which the $\mathrm{P}$ and $\mathrm{C}$ films are obtained, respectively. 


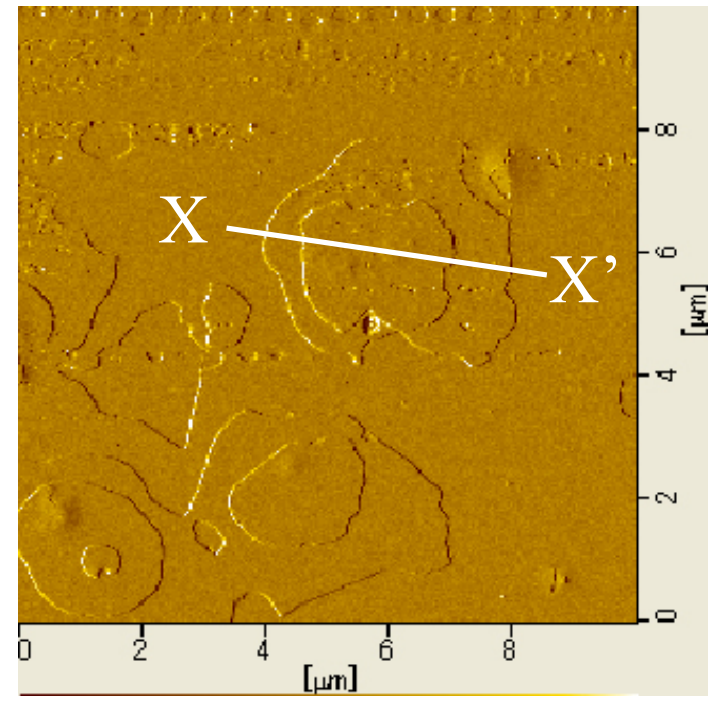

(a)

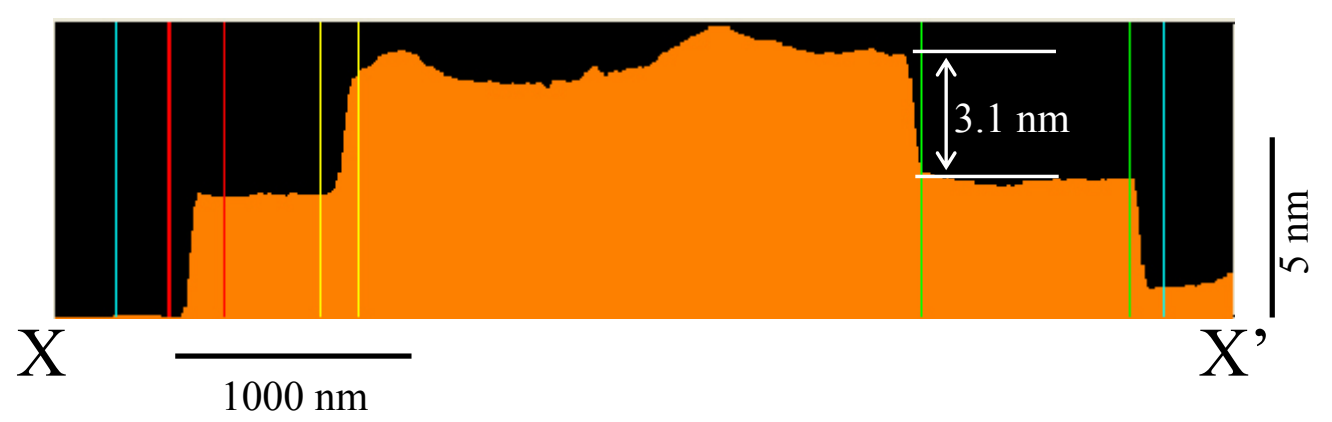

(b)

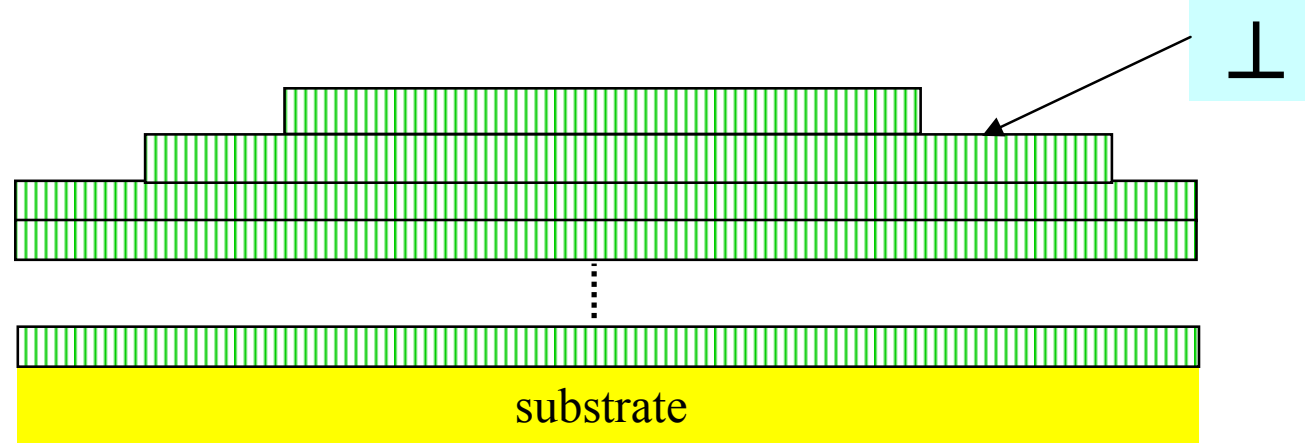

(c)

Figure 5 Surface morphology of C23 P film evaporated on SI substrate: (a) SPM (DFM) image, (b) cross-sectional image along X-X' line in SPM image, and (c) schematic of $P$ film structure predicted from SPM image. 


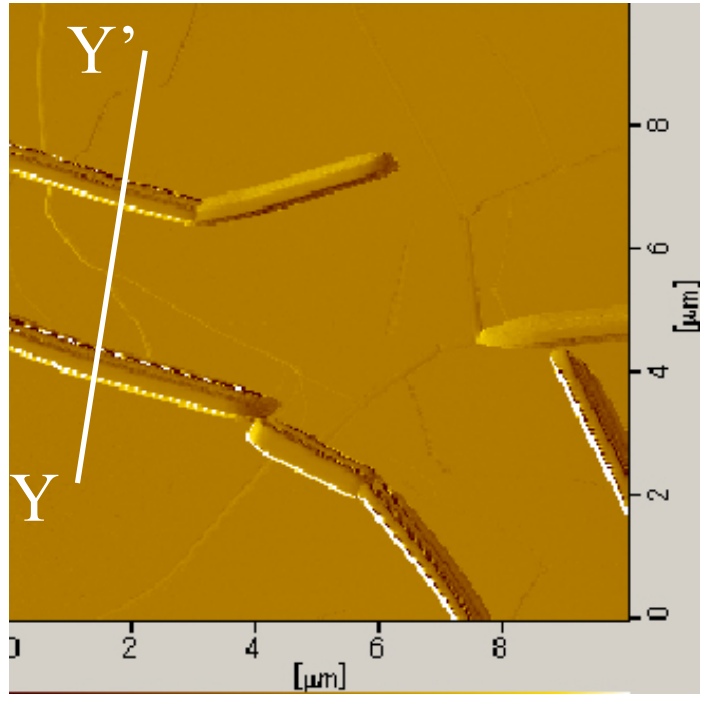

(a)
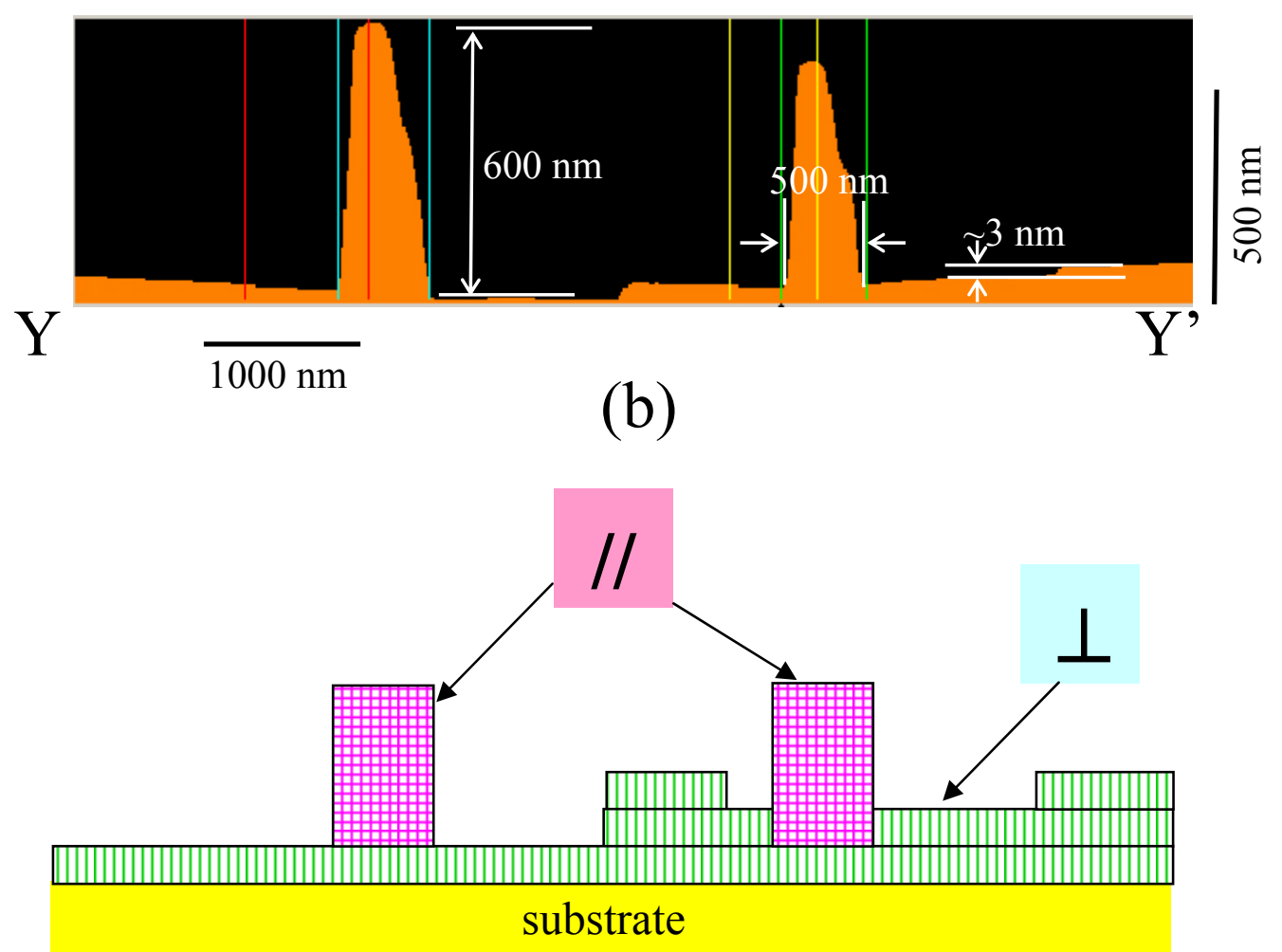

Figure 6 Surface morphology of C23 C(Filh evaporated on SI substrate: (a) SPM (DFM) image, (b) cross-sectional image along the X-X' line in the SPM image and (c): schematic of $P$ film structure predicted from the SPM image. 


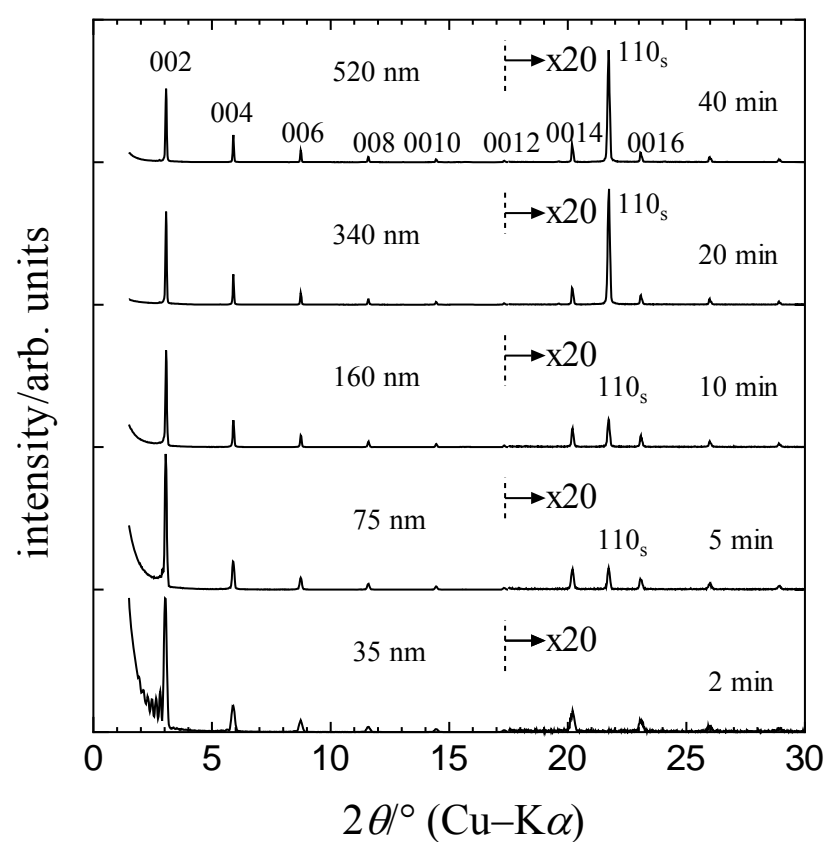

(a)

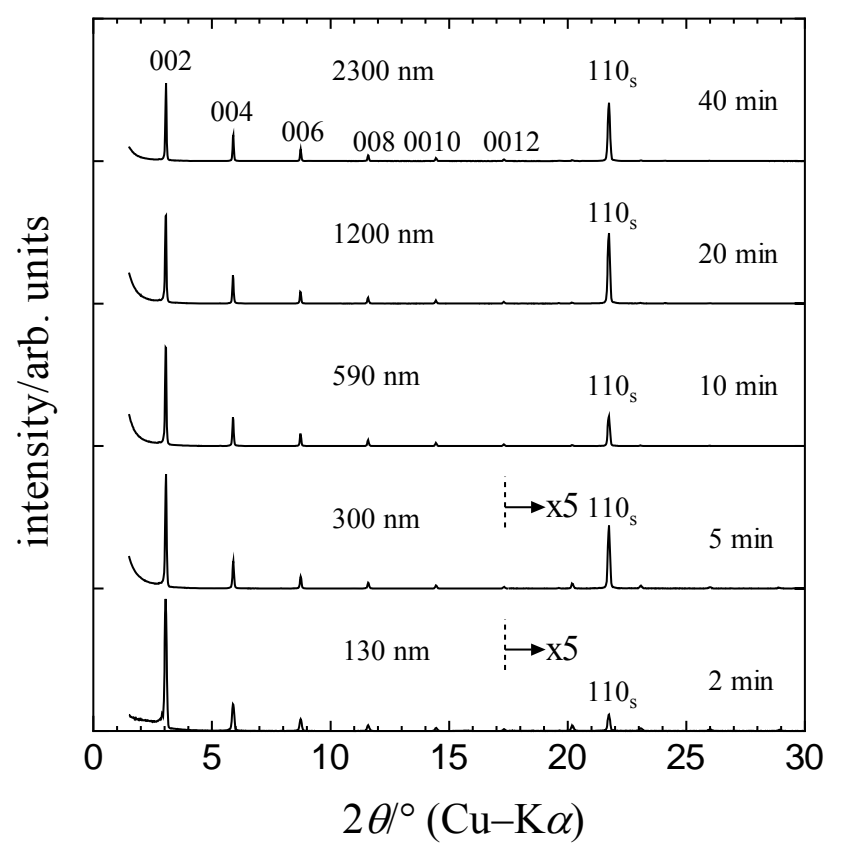

(b)

Figure 7 Deposition time dependences of X-ray diffraction profiles of C23 evaporated films at substrate temperature of $25^{\circ} \mathrm{C}$ and evaporation rates of (a) $25 \mathrm{mg} / \mathrm{h}$ and (b) 42 $\mathrm{mg} / \mathrm{h}$. 

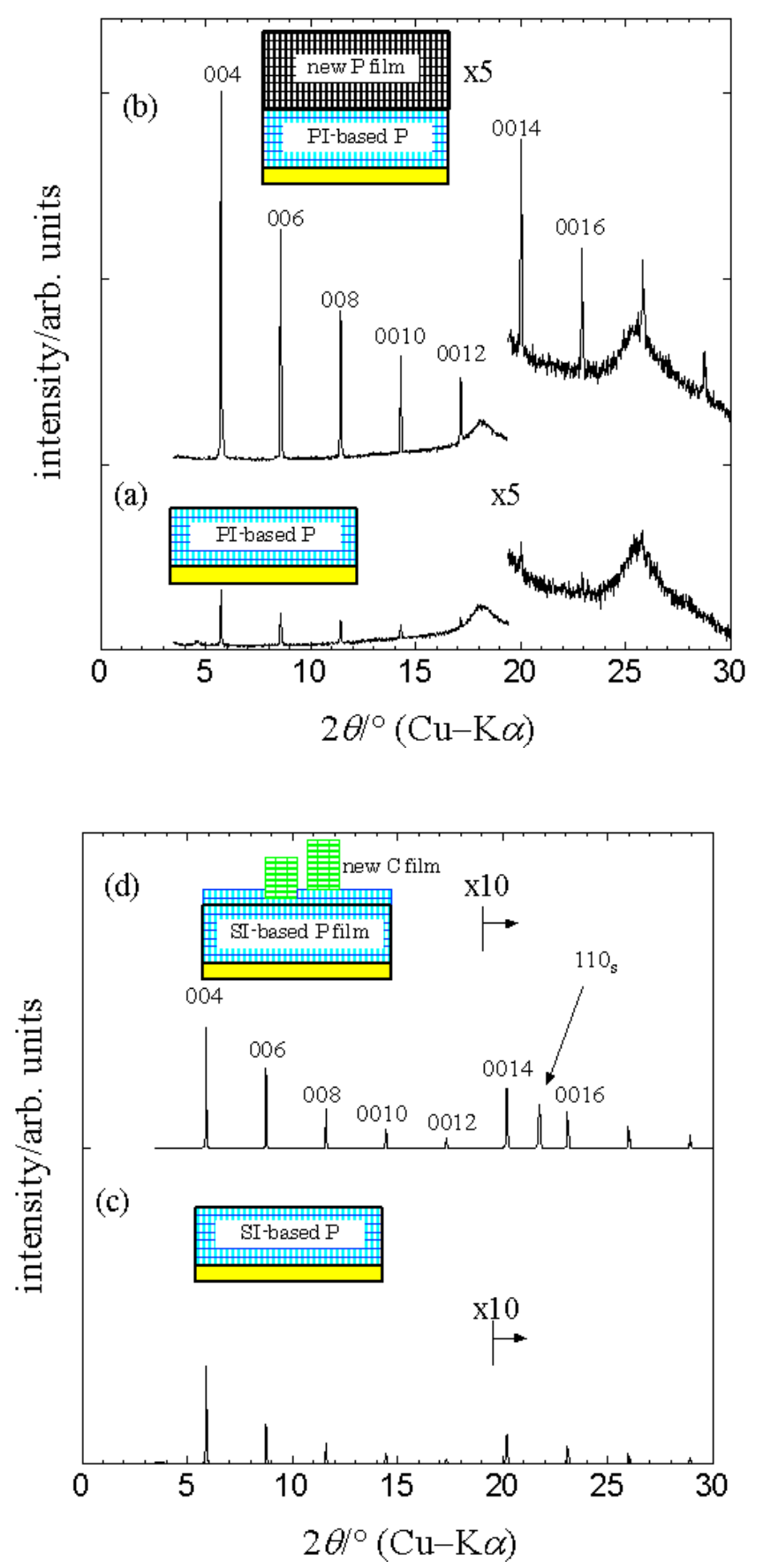

Figure 8 X-ray diffraction profiles of (a) base $\mathrm{P}$ film on PI substrate, (b) C23 evaporated film deposited on PI-based P film, (c) base P film on SI substrate, and (d) C23 evaporated film deposited on SI-based P film. 